\title{
Molecular biology and immunology of gastric cancer peritoneal metastasis
}

\author{
Xiaodan Yao, Jaffer A. Ajani, Shumei Song \\ Department of Gastrointestinal Medical Oncology, The University of Texas MD Anderson Cancer Center, Houston, TX, USA \\ Contributions: (I) Conception and design: S Song, X Yao; (II) Administrative support: None; (III) Provision of study materials or patients: None; (IV) \\ Collection and assembly of data: None; (V) Data analysis and interpretation: None; (VI) Manuscript writing: All authors; (VII) Final approval of \\ manuscript: All authors. \\ Correspondence to: Shumei Song, MD, PhD; Jaffer A. Ajani, MD. Department of Gastrointestinal Medical Oncology, Unit 426, The University of \\ Texas MD Anderson Cancer Center, 1515 Holcombe Boulevard, Houston, TX 77030-4009, USA. \\ Email: ssong@mdanderson.org; jajani@mdanderson.org.
}

\begin{abstract}
Peritoneal metastases occur in 55-60\% of patients with gastric cancer (GC) and are associated with a $2 \% 5$-year overall survival rate. There are limited treatment options for these patients, and no targeted therapy or immunotherapy is available. Rational therapeutic targets remain to be found. In this review, we present the published literature and our own recent experience in molecular biology to identify important molecules and signaling pathways as well as cellular immunity involved in the peritoneal metastasis of GC. We also suggest potential novel strategies for improving the outcomes of GC patients with peritoneal metastasis.
\end{abstract}

Keywords: Gastric cancer (GC); epithelial-mesenchymal transition (EMT); immunology; molecular biology; peritoneal carcinomatosis (PC); peritoneal metastases

Received: 11 December 2019; Accepted: 03 February 2020; Published: 05 October 2020.

doi: $10.21037 / \operatorname{tgh} .2020 .02 .08$

View this article at: http://dx.doi.org/10.21037/tgh.2020.02.08

\section{Introduction}

Gastric cancer (GC), is the fifth most commonly diagnosed cancer in the world, and over a million new cases of GC are diagnosed each year (1). According to GLOBOCAN 2019 data, GC is the third leading cause of cancer deaths worldwide, following lung and colorectal cancer in overall mortality. GC is often not found until it is at an advanced stage. Mortality from GC is higher among males. An estimated that $55-60 \%$ of GC patients develop peritoneal metastases (2). Peritoneal metastasis is life-threatening and presents an unmet need. Despite the use of multiple modalities to treat GC, including gastrectomy combined with radiation therapy, chemotherapy or targeted chemo-immune therapy, the disease often progresses, relapses, or metastasizes and has a 5 -year survival rate of less than $35 \%$ overall (3); and only $2 \%$ presence of peritoneal metastases (4).
The gastrointestinal cancers include GC, colorectal cancer, appendicular cancer, and pancreatic cancer. The peritoneum tends to be an ideal spreading ground for distant metastasis caused by epithelial cancers (5). Malignant ascites can occur as a result of gastric, colonic, pancreatic, and ovarian carcinomas. Malignant ascites has an extremely poor prognosis and lacks treatment. Novel technologies in genomics and proteomics, including singlecell RNA sequencing and cytometry by time of flight have become increasing efficient and important techniques in addressing the challenges presented by metastases. Recently, several review articles on GC have discussed the genomics of peritoneal malignancies (6), molecular carcinogenesis (7) and molecular mechanisms of peritoneal dissemination (4), clinical significance of micrometastases detected in various anatomic sites in patients with GC (8). In this review 
we present recent findings in the molecular biology and immunology of gastrointestinal peritoneal metastasis with a focus on GC.

\section{Mechanisms and important molecules involved in peritoneal metastasis}

\section{Mutations}

Genetic mutations, specific gene alterations, have been found in patients with GC. Less than $2 \% \mathrm{GC}$ cases are familial in origin. Hereditary susceptibility in GC has recently been screened. Thirty-seven percent had a first-degree relative with GC, and almost $90 \%$ had a family history of a firstand/or second-degree relative with cancer (9). Hereditary diffuse GC is inherited in an autosomal dominant manner and is caused by cadherin 1 (CDH1, i.e., E-cadherin) gene mutation. CDH1 function is frequently lost in a variety of epithelial tumors, and the loss of normal intercellular junctions promotes cancer invasion and metastases and is associated with several types of cancers including GC (10).

Most genetic mutations identified in GC are associated with changes in biological signals, such as the phosphatidylinositol 3-kinase/AKT target of the rapamycin pathway (11). Mutations in the PI3K/AKT pathway-related genes, PIK3A, PTEA, AKT3, $A K T 2$ and $A K T 1$, which are seen in a higher percentage of patients with diffuse-type GC, can affect patterns of recurrence. GC patients with PIK3CA amplifications were likely to have diffuse-type and poorly differentiated GC and peritoneal recurrences.

To identify gene clusters that plays a key role in peritoneal metastasis, one group of researchers performed comprehensive whole-genome and transcriptome sequencing analysis of matched primary GC and peritoneal metastasis from GC (12). They found 27 mutated genes, of which 19 genes (ACADL, ACAN, AKAP2, ATXN3, CCDC121, CORO1B, CRNN, DCTN1, HS6ST3, ITGAD, IQUB, IREB2, JAM2, MUC4, MUC17, PRB4, PRB1, $R P 1 L 1$, and ZNF208) were reported in Catalogue of Somatic Mutations in Cancer database and eight genes were newly described in GC (ARMC4, CCDC178, DAB1, DMBT1, PDZD2, PLIN4, PKLR, and TUBB6). Signaling pathways were analyzed for the gene mutations occurring in both primary cancer and peritoneal metastases. Six genes (CDH16, HOXA11, LOC100505875N, KX2-5, NOX4, and SFRP4) were up-regulated, and represented developmental proteins, tube morphogenesis, and homeobox.
Significant down-regulation of genes related to epithelium morphogenesis, secretion, and muscle development also was found in both primary cancer and peritoneal metastases compared with chronic gastritis.

Recently our research team characterized exome, transcriptome, and immune landscapes in peritoneal carcinomatosis (PC) cells from 43 patients with GC (13). We identified shared genomic alterations between primary GC and PC. We also demonstrated differences in genomic alterations in PC compared with primary GC, including increased frequency of $\mathrm{CDH} 1$, and TAF 1 mutations, increased proportion of 'clock-like' mutational signature, decreased levels of signatures associated with defective DNA mismatch repair and POLE mutations, and increased frequency of $6 \mathrm{q}$ loss and chromosome 19 gain. The complexity of intratumour heterogeneity of PC was revealed by analysis of the clonal and subclonal genomic architecture. Furthermore, the novel molecular 'mesenchymal-like' and 'epithelial-like' subtypes were defined by integrative clustering of genomic, transcriptomic, and immune features. The 'mesenchymal-like' subtype was associated with resistance to post-PC therapy. However, we did not find an association between the traditional histopathologybased subtypes and therapy response (13).

\section{Epithelial-mesenchymal transition (EMT)}

EMT, in which cells lose their epithelial characteristics and acquire mesenchymal features, has been associated with various tumor functions, malignant progression, tumor stemness, tumor cell migration, intravasation to the blood, metastasis, and resistance to therapy (14).

Asparaginyl endopeptidase (AEP), a lysosomal protease, potentially plays a role in EMT. AEP is over-expressed in GC and expressed at higher levels in PC than in primary GC. It is interesting to observe that the proliferative, invasive, and metastatic capacity of GC cells was inhibited and the population of sub-G1 cells was increased in AEP knocked-down nude mice. AEP knockdown was also associated with significantly decreased expression of the transcriptional factor Twist, increased expression of the epithelial marker E-cadherin, and as a contrast, decreased expression of mesenchymal markers, $\mathrm{N}$-cadherin, $\beta$-catenin, and vimentin. These findings indicated that AEP possibly promotes invasion and metastasis by modulating EMT (15). Further investigation revealed the significantly diminished phosphorylation of AKT and MAPK signaling pathways in 
AEP knocked-down GC cells.

In a mechanism study of EMT, expression of stromal fibroblast activation protein (FAP) showed a high correlation with the malignant level of clinical outcomes in GC patients, and promoted proliferation, migration, invasion, and apoptosis inhibition in a human GC cell line, SGC7901. The epithelial markers, E-cadherin and ZO-1, were down-regulated, while those proteins related to the mesenchymal markers $\mathrm{N}$-cadherin and $\mathrm{Wnt} / \beta$-catenin pathway related proteins, DKK1 and LEF-1, were upregulated. A xenograft GC nude mouse model showed that tumor burden was significantly enhanced by FAP. Therefore, stromal FAP is a potential prognostic biomarker in GC by promoting cancer progression during EMT through the $W n t / \beta$-catenin signaling pathway (16).

Directly involved in tumor metastasis is S100A4, a member of the S100 calcium binding protein family (17). In a recent investigation of mechanisms of $\mathrm{PC}$ and EMT process in GC, S100A4 expression level was found to be enhanced by TGF- $\beta$ stimulation, leading to upregulated epithelial markers and downregulated mesenchymal markers (18). In a screening of PC-related genes by a cDNA microarray, S100A4 and catenin $\beta 1$ (CTNNB1) are identified for up-regulation in highly metastatic peritoneal cancer cells (19). Overexpression of S100A4 also was associated with more adverse clinical features and a poor prognosis among GC patients in Asia (20). Furthermore, $\beta$-catenin signaling pathway cascades involved in pluripotent stem cell generation and cancer. The EMTrelated $C T N N B 1$ is mutated along with mutation of CARD11, EPHA8, ICE1, SS18, MSH3, and PTCH1. The expression of CTNNB1, and genes related to the CTNNB1 network, including CDH1, LEF1, MYC, and TCF7L2 was up-regulated in diffuse-type GC cells compared with mesenchymal stem cells $(21,22)$.

ALEX1 is a member of the Armadillo family. Its DNA was hypermethylated in the promoter region and its expression was dramatically reduced in GC tissues compared with corresponding adjacent non-tumor tissues. The lower expression of ALEX1 was associated with poor differentiation, lymph node metastasis, and shortened patient survival. ALEX1 contributed to the pleiotropic effects in GC cells, such as reduced cell proliferation, migration, and invasion in both in vitro and in vivo functional analyses. Furthermore, ALEX1 was proved to participate in EMT and attenuate thrombin-induced metastasis via the repression of Rho GTPase activation. These results suggest that ALEX1 plays a metastatic suppressive role in GC patients with PC (23).

\section{Other proteins involved in GC with $P C$}

Various proteins influence the aggression of GC progression and PC metastases (24). The protein markers for peritoneal dissemination involve invasion of gastric wall (EpCAM, E-cadherin, AnxA1 and NRAGE), outliving and proliferation (HIF-1 $\alpha$, PTEN, CAFs, CXCR4, AREG and HB-EGF), adhesion and invasion to the mesothelium (ITGAs, MELK, MMP7 and CTGF), and expansion and angiogenesis (VEGF and IRX1). These factors involve PI3K/Art, MAPK/ERK and angiogenesis signaling pathways. Additional molecules involved in gastric carcinogenesis and peritoneal metastasis are described below.

\section{Mucins}

Mucin family members are glycosylated membrane-bound proteins, produced in epithelial cells, serving functions from lubrication to cell signaling and chemical barrier formation. The roles of some mucins in GC and metastasis were recently well summarized in a review (24). Mucins are frequently engaged in an inhibitory role. Overexpression of selective mucins is correlated between clinicopathologic profiles and patient survival in the gastric carcinogenesis process. MUC4 has been involved in tumor growth and metastasis. MUC4 is activated by the transcription factor STAT3 and by Erk1/2, while MUC2 is activated through the NF-kB pathway, and both are associated with gastric or intestinal epithelial inflammation and are dependent on individual cytokines, TNF-a, IL-1b or IL-6 (25). MUC1 is overexpressed in metastatic cancers and is often used as a diagnostic marker for metastatic progression. IL-11 is reported to promote MUC1 transcription in the co-culture of cancer-associated fibroblasts with GC cells by activating STAT3 pathway, thereby enhancing the ability of GC to metastasize (26). The different mucins combined with other markers are used for identifying GC phenotypes (7).

\section{Hypoxia-inducible factors}

Hypoxia inducible factor (HIF) 1 regulates a variety of physiologic pathways, such as hematopoietic stem cell regulation, cell proliferation, survival, apoptosis, angiogenesis, glucose metabolism, and immune cell activation (27). HIF-1 $\alpha$ regulated genes was recently found to regulate procollagen-lysine 2-oxoglutarate 5-dioxygenase 2 (PLOD2) (28), and expressions levels of both HIF- 
$1 \alpha$ and PLOD2 were induced by hypoxia. In turn, these elevated levels of PLOD2 were associated with peritoneal dissemination and poor survival in GC patients. Similarly, experiments in GC cells revealed that PLOD2 induction by hypoxia associated with cell invasiveness and migration. Conversely, the invasiveness and metastatic peritoneal of those cells were suppressed after PLOD2 silencing, independently of HIF- $1 \alpha$.

HIF- $2 \alpha$ promotes migration, invasion and metastasis in GC cells both in vitro and in vivo, and overexpression of HIF-2 $\alpha$ upregulates survivin, cyclin D1, MMP2 and MMP9 (29). The cell cycle regulators cyclin D1 and cyclin E2 are highly expressed in gastric carcinoma and are associated with clinical parameters. Cyclin D, as a diagnostic biomarker, showed significantly higher sensitivity and specificity than cyclin E2. Nonetheless, the differential expression of these cyclins is an early event in gastric carcinogenesis, and may become diagnostic biomarkers for early detection of GC (30).

\section{ST6GalNAc I}

Cell-surface glycosylation is commonly modified in cancer cells. Tumor-specific polysaccharides or glycan antigens are exclusively feature of cancer cells, affecting cell-surface receptor properties, such as binding, activity, and stability. These carbohydrate antigens on cell surface can regulate cell-cell and cell-extracellular matrix adhesion or alternate cell proliferation and evasion of the immune system (31). All of glycosylation alteration is an enzyme process.

ST6GalNAc I is a sialyltransferase controlling the expression of sialyl-Tn antigen, which is overexpressed in GC, and is highly correlated with cancer metastasis. Inhibition of ST6GalNAc I on GC in vitro and in vivo in a peritoneal dissemination mouse model by knock-down of ST6GalNAc I led to suppression of cell growth, migration, invasion, and peritoneal dissemination, and prolonged the survival of a xenograft mouse model of GC. Furthermore, the ST6GalNAc regulated gene, insulin-like growth factor-1 (IGF-1), was association with the signal transducer and activator of transcription 5B (STAT5B) signaling pathways (32).

Galectin 3, belonging to the family of b-galactosidasespecific lectins, is usually highly expressed in many cancers, including GC. ST6GalNAc-I knockdown was found to restore galectin-3-binding sites on the GC cell surface along with sensitivity to chemotherapeutics. ST6GalNAc-I enzymatic activity interrupted $O$-glycan extension on galectin 3, leading to tumor cells resistance to chemotherapeutic drugs. This phenomenon highlights the need for the development of novel strategies to target galectin-3 and/or ST6GalNAc-I (33).

\section{Nicotinamide nucleotide transhydrogenase (NNT)}

NNT is a key antioxidative enzyme that can regenerate NADPH from NADH. NNT is correlated to shorter overall and disease free survivals in GC. NNT promoted tumor growth, lung metastasis and peritoneal dissemination of GC. Moreover, intratumoral injection of NNT siRNA significantly suppressed GC growth in patient-derived xenograft models. This study demonstrated crucial functional roles of NNT in redox regulation and suggested that defense of oxidative stress is also one of manners to inactivate p38MAPK pathways (34).

\section{Carcinoembryonic antigen-related cell adhesion molecule 1 (CEACAM1)}

CEACAM1 is associated with various cancers. There are 12 isoforms of CEACAM1 with diversified functions through signaling transduction, in particular, MAPK/Erk1 pathways and PI3K/Akt pathways (35). CEACAM1 expression in GC cells modulates invasiveness, ingression, lumen formation, and tumor growth. However, loss of CEACAM1 is associated with poor prognosis and peritoneal dissemination in patients with GC. Whether CEACAM1 is a suppressor or promoter for malignant phenotypes of GC remains controversial (36). Although CEACAM1 is proposed to be a ligand for TIM-3, a novel immune-checkpoint protein both are highly expressed in malignant ascites cells (our own unpublished data), the relationship of these two molecules in GC has not yet been reported.

\section{Oncostatin $M$ receptor and discoidin-domain receptor 2}

STAT3 activation and its-mediated signaling pathway are required in tumor initiation and metastasis (37). Oncostatin $\mathrm{M}$ receptor (OSMR), a member of the IL-6 receptor family, transduces Oncostatin $M$ signal to induce tumorigenesis and PC in vivo. High level of OSMR expression was found in GC tissues and was closely correlated with age, $\mathrm{T}$ category, Lauren classification, lymph node metastasis, TNM stage and worse prognosis in GC patients. OSMR knockdown significantly downregulated cell proliferation, migration, invasion, and EMT in vitro. Moreover, SP1 was found to bind to the promoter region of OSMR gene and transcriptionally upregulate OSMR expression in GC cells. Therefore, the activation of STAT3/FAK/Src signaling is necessary for the effects of OSMR, and is a potential to be 
targeted in treating GC and metastasis (38).

Mechanisms of peritoneal dissemination have been exploited in the work of Kurashige et al. (39). They established a gene-expression signature associated with peritoneal dissemination in GC by screening metastatic cell lines and 200 GC patients. The combined analysis in vitro, xenograft nude mouse model and clinical data revealed that discoidin-domain receptor 2 (DDR2) was responsible for peritoneal dissemination. A drug, dasatinib, that inhibits the kinase function of DDR2, suppressed peritoneal dissemination in vitro and in vivo.

\section{miRNA and peritoneal metastasis}

miRNA has been explored extensively in recent years in cancer research and diagnosis. Oncogenic miRNAs activate oncogenic pathways or inhibit tumor suppressive genes, while suppressive miRNAs eliminate oncogenic gene function. One miRNA can target multiple genes or pathways through posttranslational mechanisms. Low levels of tumor suppressive miRNA are associated with features of cancer aggressiveness, e.g., cancer proliferation or tumor metastasis, and high levels of tumor-suppressive miRNA reduce cancer growth in vivo and in vitro (40) (Table 1).

Genome-wide transcriptional profiling of miRNA-bases signatures has been investigated in primary GC tissue from patients with PC (41). The three miRNAs (miR-30a-5p, $-659-3 p$, and -3917 ) were significantly overexpressed in patients with PC, which was closely correlated with poor prognosis. The combination of this miRNA signature and the Bormann macroscopic type offers a potentially accurate approach for the detection of PC.

\section{Suppress-miRNAs}

Studies of suppressive miRNA suggest potential treatment strategies for GC. miR-148a was shown to reduce tumor proliferation, metastasis, invasiveness, migratory and adhesive activities of GC cells through its regulated protein-interaction network (42). In addition, miR-26a was found to directly target fibroblast growth factor 9 (FGF9) whose overexpression in miR-26a-expressing cells could rescue invasion and growth defects of miR-26a (43). Further experiments confirmed that elevated expression of miR148a and miR-26a significantly suppressed cell proliferation, migration, invasion, and colony formation, and induced apoptosis compared with a negative control group $(43,44)$. The miR-29 family acts as tumor suppressors by targeting CCND2 and matrix metalloproteinase-2 genes in GC (45). miR29a-3p downregulated hyaluronan synthase 3 (HAS3) expression and repressed proliferation and metastasis of GC (46).

miR-198 down-regulation was characterized in GC tissues. Restoration of miR-198 expression attenuated GC cell proliferation and colony formation, while inducing significant $G_{0} / G_{1}$ arrest. Furthermore, combinatory therapy with cisplatin and miR-198 induced greater anti-tumor effects than treatment with cisplatin monotherapy. Fibroblast growth factor receptor 1 (FGFR1) was indicated a direct target gene of miR-198, and FGFR1 silencing elicited a similar tumorsuppressive effect to that caused by miR-198 overexpression. Conversely, FGFR1 overexpression antagonized the antitumor effects of miR-198 overexpression. The miR-198/ FGFR1 axis therefore plays an important role in proliferation and apoptosis of GC (47).

miR-140 expression was significantly reduced in $H$. pylori positive GC. The immune checkpoint molecule PDL1 was identified as a direct target of miR140 in GC cells, and overexpression of miR140 significantly suppressed GC cell proliferation by reducing PD-L1 expression. miR-140 markedly repressed tumor growth in C57BL/6 mice. It is further confirmed that increased cytotoxic CD8+ T cells and decreased myeloid-derived suppressive and regulatory $T$ cell infiltration were closely associated with the tumorsuppressive role of miR140 in GC. In addition, the miR-140 overexpression-induced alterations were down-regulated by PD-L1 plasmid. These findings verified that miR140 exerts an anti-GC effect by targeting PD-L1 (48).

As a key regulator of cell cycle progress, cyclin E2 has been identified as the target of variety of miRNAs in cancers, including miR-30 family, as well as miR-383 in GC cells (49). miR-383 targeted cyclin E2 and negatively regulated the expression of cyclin E2 in GC. Similarly, miR-30d-5p overexpression inhibited the proliferation and motility of non-small cell lung cancer by targeting cyclin E2 (50). These studies suggested the critical function of cyclin E2 in miRNA-mediated tumorigenesis. Furthermore, miR-30a$5 \mathrm{p}$ was significantly reduced in GC tissues compared with normal gastric tissues, as well as in GC cell line AGS (51). Insulin-like growth factor 1 receptor (IGF-1R) was also identified as a target of miR-30a-5p. Furthermore, IGF$1 \mathrm{R}$ was involved in miR-30a-5p-mediated proliferation and invasion of AGS cells.

miR-338-3p level is inversely correlated with an oncogene, protein-tyrosine phosphatase 1B (PTP1B), which is involved in carcinogenesis and cancer dissemination in GC tissues. PTP1B is a target of miR-338-3p via 
Table 1 miRNAs are involved in GC progression and peritoneal metastasis

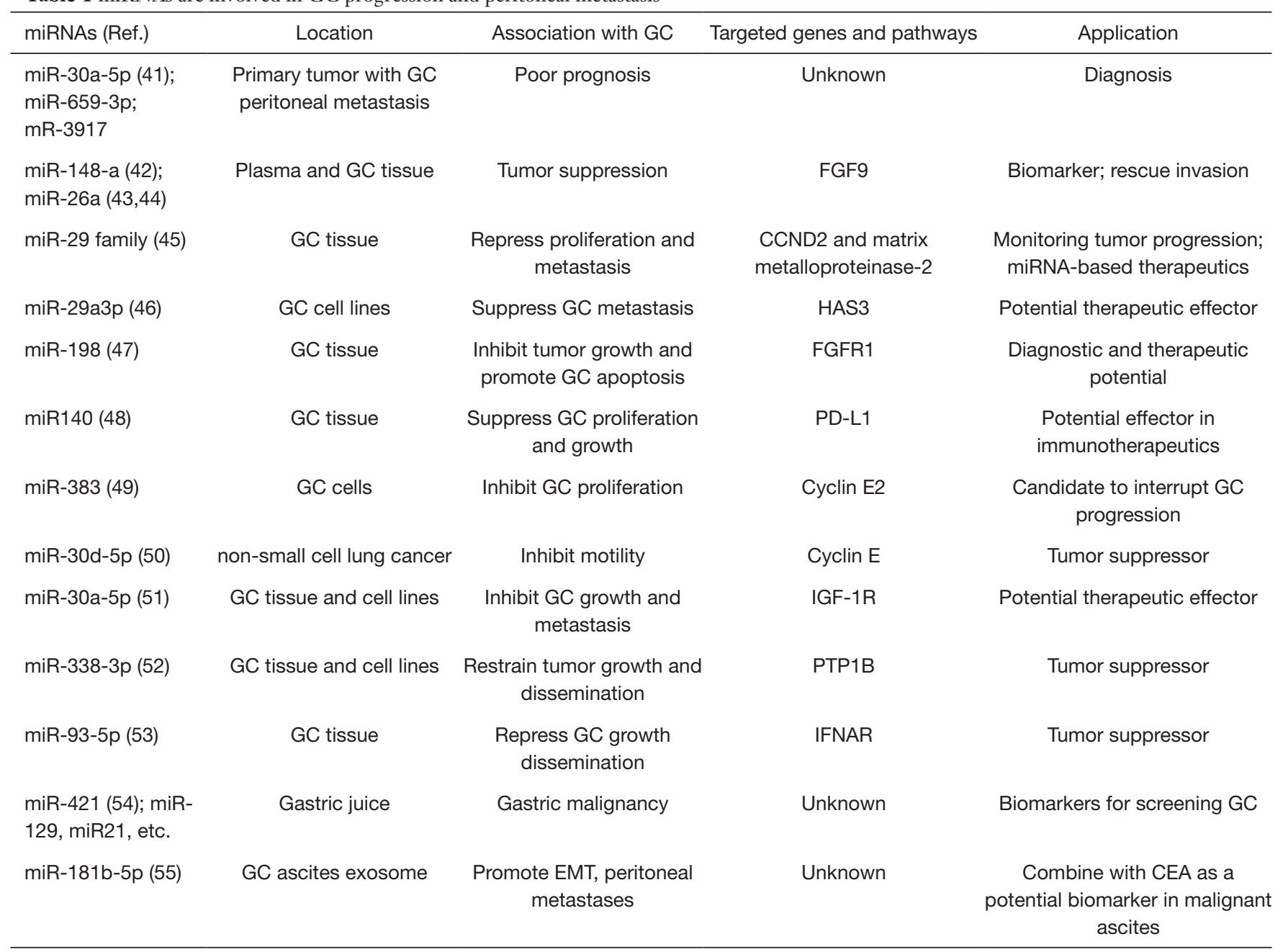

direct targeting to 3'-untranslated regions of PTP1B. Furthermore, an orthotopic xenograft model and a peritoneal dissemination model of GC also demonstrated that miR-338-3p restrained tumor growth and dissemination in vivo by targeting PTP1B. These results provide new insights into novel molecular therapeutic targets by administration of miR-338-3p to suppress PTP1B oncogene in GC (52).

\section{Onco-miRNAs}

miR-93-5p expression was increased in GC tissues compared with adjacent normal tissues and its overexpression was correlated with distant metastasis and poor survival in GC patients. miR-93-5p knockdown restricted the migration, invasion and proliferation of GC cells in vitro and in vivo.
Furthermore, miR-93-5p significantly decreased IFNAR1 expression in GC cells. IFNAR1 is a direct target of miR93-5p. IFNAR1 knockdown promoted migration and invasion of GC cell. As a consistent result, miR-93-5p overexpression rescued migration and invasion of GC cells. In sight of acting mechanism, miR-93-5p-IFNAR1 axis increased MMP9 expression via activation of STAT3 pathway to promote GC metastasis in GC (53).

\section{miRNA and exosomal miRNA as biomarkers}

miRNA profiles display the potential for miRNA as biomarkers in various gastric diseases, including Helicobacter pylori infection, chronic gastritis, and intestinal/gastric types of GC at various stages (56). Body fluids such as peripheral blood, urine or saliva, stomach wash or gastric juice could be 
a source of specific biomarkers for screening and diagnosis in GC. Circulating molecules such as miRNA, along noncoding RNAs, and circular RNAs hold promise in the development of new strategies for early diagnosis (57). Analysis of circulating miRNA can provide important information on GC prognosis. miRNAs in gastric juice could represent a cogent alternative to screening for GC by biopsy, as the gastric juice miRNA test is reliable and reproducible. MiR-421, miR-129, miR-21, miR-106a, and miR-133a were investigated in the gastric juice of patients (54).

Exosomes derived from malignant ascites from GC patients and GC cells have been investigated as potential biomarkers for GC. Exosomal miR-181b-5p combined with carcinoembryonic antigen (CEA) level was found to distinguish between non-malignant and GC-ascites (55). Exosome miRNAs are highly expressed in plasma and PC, and play a pivotal role in cancer progression (58). Twentynine differentially expressed exosomal miRNAs were found to up-regulate of EMT signaling and initiate peritoneal metastasis in vitro and in vivo. Furthermore, 12 miRNAs including miR-760, miR-6821-5p, etc. were predicted to their target genes, which are involved in TGF- $\beta$, Wnt, Notch and PI3K-AKT signaling pathways and play roles in GC patients with peritoneal metastasis. These miRNAtranscript interactions could provide a novel perspective of mechanisms of peritoneal dissemination in GC. The exosomal miRNAs thus present potential biomarkers or targets for GC patients with peritoneal metastasis.

\section{Immunology of peritoneal dissemination in GC}

Innate and adaptive immune cells in peritoneal metastasis from $G C$

Macrophages (M $\varphi$ ), neutrophils, dendritic cells (DCs) and natural killer $(\mathrm{NK})$ cells participate in innate immunity via direct defense against invading pathogens and cancer cells, and furthermore initiate adaptive immune systems. $T$ and B lymphocytes function act as major "soldiers' in adaptive responses through interaction with DCs, monocytes, and $M \varphi$. Overall, the basic functional and phenotypical characteristics of these mononuclear phagocytes are both distinct and, often, overlapping (59) (Figure 1).

\section{DCs}

DCs are the most powerful professional antigen-presenting cells at the interface between innate and adaptive immunity with the ability to activate many effector cells $(\mathrm{M} \varphi, \mathrm{NK}$,
$\mathrm{T}$, and $\mathrm{B}$ cells). DCs also can induce antigen-specific $\mathrm{T}$ cell immunity. DCs were recently reported to show an antitumor function as an activator of resting or naive $\mathrm{T}$ cells in GC in vitro (60). The sphere-forming cells were isolated and identified from the patients with GC, and then co-cultured with autologous T-lymphocytes for educating and boosting specific anti-tumor lymphocytes. IFN- $\gamma$ gene expression in these lymphocytes was significantly higher compared with the control. The cytotoxic activity of T-lymphocytes primed with antigens of sphere-forming cells was also significantly higher than that of normaltissue antigens and mock DCs. Therefore, DCs are pulsed with antigens of sphere-forming cells due to the lysis of GC stem cells by primed T-lymphocytes in vitro to exclude the potential for adverse autoimmune events or other side effects. This strategy could be used as a therapeutic vaccination against autologous cancer stem cells in GC patients in the future.

\section{$M \varphi$ and enriched $M 2 M \varphi$ in $\mathbf{P C}$}

$M \varphi$ are important in phagocytosis, antigen presenting and producing cytokines and growth factors. There are limited strategies based on patient immunity for treating peritoneal dissemination of malignancies in the abdominal cavity. Since immune cells such as $M \varphi$ and lymphocytes are present in the greater omentum and lymph nodes, the activation of immune cells would be a promising strategy for treatment of PC. A decade ago, the recognition by Toll-like receptor ligand, on antigen-presenting cells such as $M \varphi$, of singlestranded oligonucleotides containing $\mathrm{CpG}$ dinucleotides (CpG DNA), was reported to stimulate Th-1-type immune responses, and the use of cationic liposomes for CpG DNA delivery was described as an efficient way to boost immune cells for treatment of peritoneal dissemination (61).

From the evolutionary point of view, mammalian organisms develop immune systems to defend against pathogens and survive in living environments. However, cancer cells either inherently or causatively develop strategies to escape immune surveillance by targeting or hijacking the immune system to assist their abnormal growth. For example, in response to micro-environmental signals, $M \varphi$ can polarize from tumor suppressing $M 1$ $M \varphi$ into tumor promoting $M 2 M \varphi$. M1 M $\varphi$ contribute to antitumoral immunity and inflammatory response by producing pro-inflammatory cytokines, such as IL-6, IL-8, IL-12 and TNF $\alpha$ (62), while a endogenous neuropeptide, Met-enkephalin (MENK), exerts antitumor activity by 


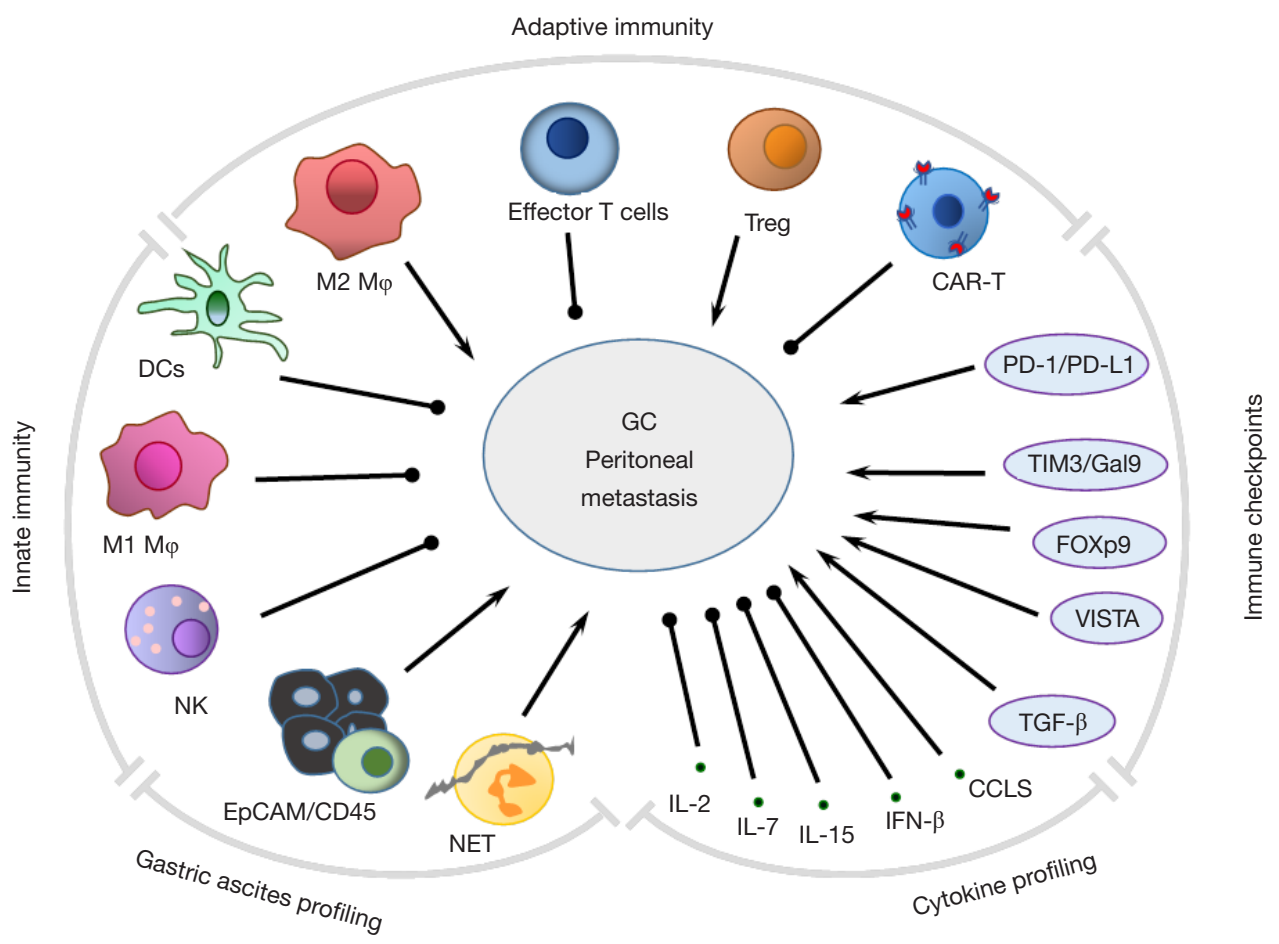

Figure 1 Immunological outline of peritoneal dissemination in GC. DCs, M $\varphi$ and NK cells are the innate immune cells directly defend against cancer. DCs and M $\varphi$ interact with T cells by further presenting GC antigens to different types of T cells and inducing or disabling immune responses to GC. While GC cells program T cells and M2 M $\varphi$ to express immune checkpoint molecules for making effector T cells into exhaustion and modifying Treg to suppress immunity in tumor-infiltrating environments. Immune cells normally secret cytokines to restrict cancer growth. However, the suppressive T cells are unable to produce these cytokines. $\longrightarrow$, enhanced peritoneal metastasis in GC;

-, inhibited peritoneal metastasis in GC. GC, gastric cancer.

reversing tumor-associated $\mathrm{M} \varphi$ polarization from $\mathrm{M} 2$ to M1 phenotype (63).

Recently, studies have indicated that M2-type $M \varphi$ resembling tumor-associated $M \varphi$ are a potential independent prognostic factor for GC (62). Tumorassociated $\mathrm{M} \varphi$ displays a M2-like phenotype with major roles in progression of solid tumors, as $\mathrm{M} 2 \mathrm{M} \varphi$ promotes tumor growth by (I) stimulating tumor cell proliferation via secretion of angiogenetic factors; (II) inducing transient openings in tumor neovessels that allow malignant cells to enter the bloodstream; (III) promoting metastatic dissemination of solid tumors; (IV) contributing to tumor relapse with multiplexing its number after chemotherapy and, limiting the efficacy of chemotherapies; and (V) supporting immune suppressive tumor microenvironment mediated by PD-1 and CTLA-4 (64). M2 tumor-associated $\mathrm{M} \varphi$ are derived from circulating peripheral blood monocytes that may already express M2-associated markers, such as CD163 and CD206, which are further upregulated upon extravasation of the cells at the tumor location and/or by exposure to factors in the perivascular tumor microenvironments (65). Recently, we have demonstrated that $\mathrm{M} 2$-like $\mathrm{M} \varphi$ infiltration was tightly associated with PD-L1 expression in gastric adenocarcinoma cells, suggesting that $M \varphi$ is a potential therapeutic target in the disease (66). In addition, in more than 200 cases, we found that a high proportion of M2 M $\varphi(\mathrm{CD} 163+)$ are enriched in malignant ascites cells (unpublished data). Therefore, targeting $\mathrm{M} 2 \mathrm{M} \varphi$ in GC peritoneal metastasis might provide novel therapeutic strategies for GC patients with PC.

\section{NK cells}

NK cells often lack antigen specific cell surface receptors, but contain granules, perforin and proteinases, e.g., granzyme $\mathrm{B}$, inducing cytolytic granule-mediated 
apoptosis or osmotic cell lysis to target cells. NK cells express activating receptors, such as NKG2D and natural cytotoxicity Receptor family (NKp30, NKp46 and NKp44) and recognize many tumor types (67). NK cells also interact with DCs, $M \varphi$ and T cells and contribute to anti-tumor adaptive immune responses by secreting cytokines, such as IL-2, IL-12, IL-15, IL-18 and CCR5.

NK cells contribute to immune surveillance of primary tumor formation, tumor recurrence and metastasis dysfunction in mouse models and human GC patients (68). However, NK cells display a suppressive phenotype with fewer number of activating receptors and higher expression of PD-1 in GC patients. In addition, NK cells secret fewer kinds of cytokines and showed decreased ability to release perforins and granzymes. Meanwhile, GC cells reduce the expression of NKG2D ligands, MICA/B and ULBP, and NKp30 ligand, B7H6, to escape NK cell-mediated innate immunity. GC cells can also release some cytokines, such as IL-10, TGF- $\beta$, and PGE2, recruit myeloid-derived suppressor cells, and regulatory $\mathrm{T}$ (Treg) cells to suppress NK cell activity.

Two reports provide approach for improving the immunogenicity or susceptibility of GC peritoneal metastasis to NK cells. One method is the use of gene therapy with adenovirus vector vehicle intercellular adhesion molecule (ICAM)-2, which was injected into mice bearing peritoneal dissemination of scirrhous gastric carcinoma (69). The mice survived longer significantly, and many NK cells filtrated the peritoneal metastatic lesions, suggesting that ICAM-2 transfection might be an efficient strategy of gene therapy for peritoneal metastasis from GC. Another method targets cancerous IgG either by transfecting GC cells with cancerous Ig G heavy chain siRNA to enhance antibody-dependent cellular cytotoxicity induced by an EGFR antibody and therefore inhibits GC cell growth (70).

\section{Treg cells}

Treg cells are subpopulation of $\mathrm{T}$ cells with typical CD4, Foxp3 and CD25 as cell type markers. Tregs are immunosuppressive and generally downregulate effector $\mathrm{T}$ cells, therefore are thought to contribute to tumor progression (71). The accumulation of Tregs in adoptive cell therapy has been investigated in peritoneal metastasis from GC (72). It is found that Treg cells were significantly more frequent in malignant ascites lymphocytes, compared with the peripheral blood of patients. Furthermore, arsenic trioxide $\left(\mathrm{As}_{2} \mathrm{O}_{3}\right)$ treatment significantly reduced Treg cell numbers and FOXP3 mRNA levels in vitro. IFN- $\gamma$ levels were increased, whereas IL-10 and TGF- $\beta$ levels were decreased by $\mathrm{As}_{2} \mathrm{O}_{3}$ in the supernatant of tumor-infiltrating lymphocytes derived from ascites. Thus, $\mathrm{As}_{2} \mathrm{O}_{3}$ may induce selective depletion and eliminate immunosuppressive function of Treg cells by promoting apoptosis of CD4+ T lymphocytes, enhancing the cytotoxic activity of tumorinfiltrating lymphocytes.

\section{Chimeric antigen receptor $T$ cells}

Chimeric antigen receptor (CAR)- $\mathrm{T}$ cell-based cancer immunotherapy is a promising new alternative for the treatment of relapsed lymphoblastic leukemia, multiple myeloma and lymphoma by inducing the direct destruction of cancers. Specific CAR-T cells are genetically engineered from patient $\mathrm{T}$ cells and can secrete cytokines, produce specific molecules and exert potent cytotoxicity against a wide range of cancer cells. The application of CAR-T cell technology in solid tumor therapy is a current research "hotspot" (73).

GC cells expressing NKG2DLs are highly susceptible to destruction by NK cells. Targeting NKG2DLs with engineered $\mathrm{T}$ cells expressing a chimeric NKG2D (chNKG2D) has been shown to induce tumor elimination and long-term tumor-free survival in mouse models (74). Furthermore, the extracellular domain of the NKG2D was constructed in human peripheral blood $\mathrm{T}$ cells containing intracellular domain of CD28 in tandem with CD3zeta to enhance T-cell activation. This chNKG2D CAR expressed on the $T$ cells allows the $T$ cells to specifically recognize NKG2DLs on the cancer cell surface of human GC cell lines and primary ascites-derived GC cells expressing surface NKG2DLs. Thus, chNKG2D receptor-modified $\mathrm{T}$ cells represent a potential therapeutic strategy for NKG2DL-expressing GC with peritoneal metastasis.

A new type of CAR-T cells is needed to make breakthroughs in the solid tumor treatment "bottleneck". A new potential target in GC is human trophoblast cell surface antigen 2 (Trop2), which is often expressed in various epithelial tissue tumors, including GC cells, and promotes malignant biological behavior including lymph node and distant metastasis (75). Similarly, PD-L1 is expressed at significantly higher levels in GC tissues, and the lymphoid tissues of GC metastasis and is related to a poor survival prognosis (76). The bi-specific Trop2/PD-L1 third generation CAR-T method was developed recently (77). The constructed CAR-T lentivirus contains an anti-Trop2 scFv sequence (heavy chain variable Trop2 VH region- 
Linker-Trop2 light chain variable region), and an antiPD-L1 scFv sequence (PD-L1 heavy chain variable regionLinker and PD-L1 light chain variable region), and is tandemly connected to CD8T transmembrane hinge, transmembrane domains of CD28 and CD137, and the cytoplasmic component of $\mathrm{CD} 3 \zeta$ chain. This CAR structure on T-cells can bind to Trop2 or the PD-L1 extramembrane domain of GC cells. The killing ability of bi-specific Trop2/ PD-L1 CAR-T cells is greater than that of mono-specific CAR-T cells (Trop2 CAR-T and PD-L1 CAR-T) and the independent control group. The levels of the cytokines, IFN- $\gamma$ and IL-2, released by bi-specific Trop2/PD-L1 CAR-T cells were the highest among all other types of CAR-T cells and the control group. Furthermore, in a xenograft model bearing human gastric tumors, bi-specific Trop2/PD-L1 CAR-T cells significantly reduced tumor growth compared with mono-specific CAT cells and the control group. This study provides a novel strategy for the clinical application of bi-specific CAR-T cell technology targeting Trop2/PD-L1 and checkpoint blockade in solid tumors, and shows the potential for high efficiency in treating GC.

\section{Immune checkpoint molecules in GC peritoneal metastasis}

Immunological checkpoint inhibitors have effects on various advanced cancers. However, their basic research and clinical trials have not yet established in peritoneal metastasis from GC. In our work with multiplex profiling of peritoneal metastasis (13), we identified not only gene mutation signatures, but also immune profiles for two main groups of PC specimens: the T-cell 'exclusive' and T- cell 'exhausted' subtypes. It is interesting to show high levels of immune checkpoints, T-cell immunoglobulin and mucin domaincontaining protein 3, (TIM-3, also called HAVCR2) and its ligand galectin-9, V-domain Ig suppressor of T-cell activation (VISTA) and TGF- $\beta 1$ in the T-cell 'exhausted' subtype, providing potential novel therapeutic immune targets. Thus, molecular classification may be more meaningful in stratification of PCs for therapy decisions.

\section{PD-L1}

Recently, the U.S. Food and Drug Administration approved pembrolizumab for patients with PD-L1-positive metastatic GC. In a study of 225 patients with advanced GC, PD-L1 expression on tumor cells, and on immune cells, mismatch repair-deficiency, and Epstein-Barr virus positivity were identified (78). PD-L1 expression in tumor cells was more frequently observed in patients with deficient mismatch repair (PIK3CA and KRAS mutations), while PD-L1 on immune cells was associated with EpsteinBarr virus positivity and lymph-node metastasis. PDL1 expression on either immune cells or tumor cells was less frequently observed in patients with peritoneal metastasis. Significant association was no or less observed between PD-L1 expression and receptor tyrosine kinase expression or the presence of other gene alterations. PD-L1 expression on either tumor cells or immune cells was not a prognostic factor. These findings suggest that immune checkpoint molecules, PD-1 and its ligand, are unlikely specific suppressive molecules among potential therapeutic targets in peritoneal metastasis from GC. Nevertheless, immunotherapeutic strategy of targeting multiple immune checkpoints is highly challenged to decide based on individual patients' genetic and immune status in detail.

The tumor immune microenvironment is changed after neoadjuvant chemotherapy in locally advanced GC. A panel of tumor-infiltrating immune cells and checkpoint molecules of lymphocytes was investigated in 60 patients with advanced GC (79). The overall expression levels of CD4, CD8, PD1, PD-L1 and TIM-3 were significantly increased at post-neoadjuvant chemotherapy, compared with prior to treatment. Individually, as contrast of increased expression of the markers in majority of patients, minorities of patients had decreased expression of these markers plus FOXP3. Alterations in expression between pre- and post-neoadjuvant chemotherapy of PD-1, PD-L1 and TIM-3 showed strongly positive pairwise correlations with each other. Multivariate analysis demonstrated that high levels of CD8, PD-1, and PD-L1 at-post neoadjuvant chemotherapy were beneficial prognostic factors for overall survival. The overall increased expression of checkpoint molecules and infiltration of immune cells might indicate a dynamic bi-directional shift in the tumor microenvironment during neoadjuvant chemotherapy in GC. These data provide the possibility of applying chemotherapy combined with immunotherapy or even dual checkpoint blockage in locally advanced GC.

\section{TIM-3 and galactin-9}

TIM- 3 is encoded by the HAVCR2 gene and expressed more or less on all of immune cells as an immune checkpoint receptor. TIM-3 level was significantly upregulated in monocytes of peripheral blood mononuclear cells from GC patients compared with those from healthy controls (80). The upregulated TIM-3 levels were correlated to depth of 
tumor invasion, tumor lymph node metastasis, and advanced clinical stages of GC. In addition, the mouse model revealed that TIM-3 level on monocytes/M $\mathrm{M}$ was associated with xenograft formation and growth rate. Furthermore, TIM3/Gal-9 signal significantly stimulated monocytes to secrete IL-6, IL-8, and IL-10 in presence of LPS. TIM-3 expressed on monocyte/M $\mathrm{M}$ might be involved in regulation of $\mathrm{M} \varphi$ function and contributed to an important mechanism in GC progression.

Galectin-9 (Gal-9) is identified as a ligand of TIM3. Its expression is also related to tumor cell adhesion or metastasis in solid tumors. The TIM-3/Gal-9 axis may play a role in promoting $\mathrm{T}$ cell exhaustion (81). Higher expression of Gal-9 was demonstrated in GC and subcellular localization in the extra cellular area and cytoplasm of tumor cells (82). In the same study, TIM3 was expressed mainly in immune cells, with minimal expression in GC cells. Expression of TIM3 and Gal-9 as well as Foxp3+ $\mathrm{T}$ cell density was reversely correlated to the overall survival rate in patients. The TIM-3/Gal-9 signal may contribute toward a suppressive tumor microenvironment via Treg cell activation. Gal-9 either promotes or inhibits tumor activity, depending on its interactions with its ligands on T cells, antigen-presenting cells, or tumor cells. Gal-9 functions that are associated with GC peritoneal metastasis and could be combined with TIM-3 checkpoint immunotherapy have not been reported and should be exploited in the future.

\section{Cytokine profiles in peritoneal metastasis}

\section{IL-2}

Immune cells secreted cytokines in response to pathogenic inflammation, infection, and cancers. IL-2 is a secreted mediator that is important for the proliferation of $\mathrm{T}$ and B lymphocytes, as well as for activation of cytotoxic T lymphocytes, helper T lymphocytes, NK cells, and lymphokine-activated killer cells (83). IL-2 administration in some tumor models has been shown to promote antitumor immunity, in which IL-2 is presumed to alleviate anergic blockage of $\mathrm{T}$ cells (84). CD80, as a ligand of CD28, plays a key role in inducing cell-mediated immune responses, and as result of co-expression of CD80 and IL-2 in T cells, tumorigenicity is decreased in some types of malignant tumors (85). Many tumors, including GC, have decreased expression of CD80, resulting in the failure of immune recognition. IL-2 addition combined with adenovirus-expressing CD80 significantly increased the activated cytotoxicity of mononuclear cells in peritoneal metastasis from GC (86). In vivo survival of mice with intraperitoneal tumor was longest in IL-2 addition plus CD80 overexpression. These results confirmed that effects of IL-2 contribute to improving CD80 therapy in peritoneal metastasis of GC. Strikingly, IL-2 is reported to rescue T cells from PD-1-mediated effects by direct activation of Akt via STAT5 and circumvent the PD-1 interference (87). Beside IL-2, other cytokines, such as IL-7 and IL-15, are also found to abate the inhibitory effects of PD-1 ligation through activation of STAT5 (88).

\section{Interferon-beta (IFN- $\beta$ )}

IFN- $\beta$ has been used to treat several cancers in preclinical and clinical research. The essential nature of interferonactivated immune signaling and its role in stimulation of immune effector cell functions for tumor prevention or eradication has been established in murine models. Novel engineered forms of human and mouse IFN- $\beta$ have been developed, named PEG-hIFN- $\beta$ and PEG-mIFN- $\beta$, in which IFN- $\beta$ is conjugated with a polyethylene glycol (89). These IFN- $\beta$ molecules retained anti-viral potency similar to natural IFN- $\beta$ in vitro and manifested improved pharmacokinetics in vivo. Interestingly, PEG-hIFN- $\beta$ directly suppressed VEGF165-induced hyperpermeability in human vascular endothelial cells. PEG-mIFN- $\beta$ significantly downregulated the accumulation of ascites fluid and vascular permeability of the peritoneal membrane in xenograft mouse models of ovarian cancer and GC. PEG-mIFN- $\beta$ enhanced expression of a number of cell adhesion-related molecules in vitro. These findings show the long-acting PEGylated hIFN- $\beta$ in maintaining vascular integrity, and provide a curative tool for efficient treatment of malignant ascites.

\section{CCL5}

Chemokine CCL5 is a demonstrated biomarker for peritoneal metastasis in patient with GC and a predictive factor for a poorer outcome. The concentrations of serum CCL5 were increased in GC patients compared with health controls, and correlated with peritoneal metastasis, more invasive histological types and more advanced disease stages. These phenomena suggest CCL5's role in the progression of peritoneal metastasis from GC (90).

\section{TGF- $\beta$}

Cytokine TGF- $\beta$ has long been studied for in inflammation and tumorigenesis. Alteration of the TGF- $\beta$ signaling pathway is associated with liver and gastrointestinal cancers. 
The function of TGF- $\beta$ is context dependent; it helps maintain tissue hemostasis by regulating cellular growth, differentiation, and survival, controls early-stage tumors by promoting cell cycle arrest, and apoptosis; and in allows for tumor invasion and metastasis at advanced stages (91).

While TGF- $\beta$ functions as a tumor suppressor in the gastrointestinal tract, it can also function as a tumor promoter. These dual effects of TGF- $\beta$ are a major barrier for the development of drugs targeting this pathway, and few drugs targeting the TGF- $\beta$ pathway have proven effective in clinical trials. Therefore, identifying novel strategies that target specific components of this pathway will lead to improved outcomes for lethal liver and gastrointestinal cancers (92). Greatly increased expression of TGF- $\beta 1$ was elucidated in the co-culture of both gastric tumor cells and mesothelial cells, compared with individual culture conditions. Serum-free conditioned media from human GC cells profoundly induced extracellular matrix expression in vitro and in vivo, and tumorigenicity in mice with peritoneal fibrosis was greater than in mice with normal peritoneum. TGF- $\beta 1$ blockage by peptide P17 partially alleviated development of peritoneal metastasis. These results indicated that TGF- $\beta 1$ has an important role in induction of peritoneal fibrosis upon interaction of GC with peritoneal fibrosis, which in turn affects dissemination of GC (93).

\section{Conclusions}

Existing research has verified broader and deeper insight into mechanisms of GC peritoneal metastasis in molecular biology and immunology. However, we are still in the middle of fully understanding GC progression and metastasis. Identifying more molecules and their interactions involved in the various signaling pathways of GC with PC in detail will lead to novel strategies to target key components of GC peritoneal metastasis and other types of GC and improve specific therapeutics in the clinic.

\section{Acknowledgments}

We appreciate Sarah Branson, a scientific editor from Scientific Publications, Research Medical Library at The University of Texas MD Anderson Cancer Center, for her excellent editing of this manuscript.

Funding: This work was supported by Public Health Service Grant DF56338, which supports the Texas Medical Center Digestive Diseases Center (S Song); an MD Anderson
Institutional Research Grant (3-0026317 to S Song); and grants from Department of Defense (CA160433 and CA170906 to S Song and CA160445 to JA Ajani); and the National Institutes of Health (CA129906, CA138671, and CA172741 to JA Ajani).

\section{Footnote}

Conflicts of Interest: JAJ serves as an unpaid editorial board member of Translational of Gastroenterology and Hepatology from Apr 2019 to Mar 2021. The other authors have no conflicts of interest to declare.

Ethical Statement: The authors are accountable for all aspects of the work in ensuring that questions related to the accuracy or integrity of any part of the work are appropriately investigated and resolved.

Open Access Statement: This is an Open Access article distributed in accordance with the Creative Commons Attribution-NonCommercial-NoDerivs 4.0 International License (CC BY-NC-ND 4.0), which permits the noncommercial replication and distribution of the article with the strict proviso that no changes or edits are made and the original work is properly cited (including links to both the formal publication through the relevant DOI and the license). See: https://creativecommons.org/licenses/by-nc$\mathrm{nd} / 4.0 \%$.

\section{References}

1. Bray F, Ferlay J, Soerjomataram I, et al. Global cancer statistics 2018: GLOBOCAN estimates of incidence and mortality worldwide for 36 cancers in 185 countries. CA Cancer J Clin 2018;68:394-424.

2. Virgilio E, Balducci G, Mercantini P, et al. Preoperative gastric lavage in gastric cancer patients undergoing surgical, endoscopic or minimally invasive treatment: An oncological measure preventing peritoneal spillage of intragastric cancer cells and development of related metastases. Med Hypotheses 2018;114:30-4.

3. Virgilio E, Giarnieri E, Giovagnoli MR, et al. Gastric Cancer Cells in Peritoneal Lavage Fluid: A Systematic Review Comparing Cytological with Molecular Detection for Diagnosis of Peritoneal Metastases and Prediction of Peritoneal Recurrences. Anticancer Res 2018;38:1255-62.

4. Kanda M, Kodera Y. Molecular mechanisms of peritoneal dissemination in gastric cancer. World J Gastroenterol 
2016;22:6829-40.

5. Alberto M, Brandl A, Garg PK, et al. Pressurized intraperitoneal aerosol chemotherapy and its effect on gastric-cancer-derived peritoneal metastases: an overview. Clin Exp Metastasis 2019;36:1-14.

6. Karunasena E, Sham J, McMahon KW, et al. Genomics of Peritoneal Malignancies. Surg Oncol Clin N Am 2018;27:463-75.

7. Oue N, Sentani K, Sakamoto N, et al. Molecular carcinogenesis of gastric cancer: Lauren classification, mucin phenotype expression, and cancer stem cells. Int J Clin Oncol 2019;24:771-8.

8. Kodera Y, Nakanishi H, Ito S, et al. Clinical significance of isolated tumor cells and micrometastases in patients with gastric carcinoma. Gan To Kagaku Ryoho 2007;34:817-23.

9. Slavin T, Neuhausen SL, Rybak C, et al. Genetic Gastric Cancer Susceptibility in the International Clinical Cancer Genomics Community Research Network. Cancer Genet 2017;216-217:111-9.

10. Zeng W, Zhu J, Shan L, et al. The clinicopathological significance of CDH1 in gastric cancer: a meta-analysis and systematic review. Drug Des Devel Ther 2015;9:2149-57.

11. Wadhwa R, Song S, Lee JS, et al. Gastric cancermolecular and clinical dimensions. Nat Rev Clin Oncol 2013;10:643-55.

12. Zhang J, Huang JY, Chen YN, et al. Whole genome and transcriptome sequencing of matched primary and peritoneal metastatic gastric carcinoma. Sci Rep 2015;5:13750.

13. Wang R, Song S, Harada K, et al. Multiplex profiling of peritoneal metastases from gastric adenocarcinoma identified novel targets and molecular subtypes that predict treatment response. Gut 2020;69:18-31.

14. Nieto MA, Huang RY, Jackson RA, et al. EMT: 2016. Cell 2016;166:21-45.

15. Cui Y, Wang Y, Li H, et al. Asparaginyl endopeptidase promotes the invasion and metastasis of gastric cancer through modulating epithelial-to-mesenchymal transition and analysis of their phosphorylation signaling pathways. Oncotarget 2016;7:34356-70.

16. Liu J, Huang C, Peng C, et al. Stromal fibroblast activation protein alpha promotes gastric cancer progression via epithelial-mesenchymal transition through Wnt/ betacatenin pathway. BMC Cancer 2018;18:1099.

17. Garrett SC, Varney KM, Weber DJ, et al. S100A4, a mediator of metastasis. J Biol Chem 2006;281:677-80.

18. Li F, Shi J, Xu Z, et al. S100A4-MYH9 Axis Promote Migration and Invasion of Gastric Cancer Cells by
Inducing TGF-beta-Mediated Epithelial-Mesenchymal Transition. J Cancer 2018;9:3839-49.

19. Bai FH, Wang NJ, Wang J, et al. Screening and identification of peritoneal metastasis-related genes of gastric adenocarcinoma using a cDNA microarray. Genet Mol Res 2012;11:1682-9.

20. Ling Z, Li R. Clinicopathological and prognostic value of S100A4 expression in gastric cancer: a meta-analysis. Int J Biol Markers 2014;29:e99-111.

21. Tanabe S, Kawabata T, Aoyagi K, et al. Gene expression and pathway analysis of CTNNB1 in cancer and stem cells. World J Stem Cells 2016;8:384-95.

22. Elimova E, Wadhwa R, Shiozaki H, et al. Molecular biomarkers in gastric cancer. J Natl Compr Canc Netw 2015;13:e19-29.

23. Pang L, Li JF, Su L, et al. ALEX1, a novel tumor suppressor gene, inhibits gastric cancer metastasis via the PAR-1/Rho GTPase signaling pathway. J Gastroenterol 2018;53:71-83.

24. Machlowska J, Maciejewski R, Sitarz R. The Pattern of Signatures in Gastric Cancer Prognosis. Int J Mol Sci 2018. doi: 10.3390/ijms19061658.

25. Ponnusamy MP, Lakshmanan I, Jain M, et al. MUC4 mucin-induced epithelial to mesenchymal transition: a novel mechanism for metastasis of human ovarian cancer cells. Oncogene 2010;29:5741-54.

26. Wang X, Che X, Liu C, et al. Cancer-associated fibroblasts-stimulated interleukin-11 promotes metastasis of gastric cancer cells mediated by upregulation of MUC1. Exp Cell Res 2018;368:184-93.

27. Hayashi Y, Yokota A, Harada H, et al. Hypoxia/ pseudohypoxia-mediated activation of hypoxia-inducible factor-1alpha in cancer. Cancer Sci 2019;110:1510-7.

28. Kiyozumi Y, Iwatsuki M, Kurashige J, et al. PLOD2 as a potential regulator of peritoneal dissemination in gastric cancer. Int J Cancer 2018;143:1202-11.

29. Tong WW, Tong GH, Chen XX, et al. HIF2alpha is associated with poor prognosis and affects the expression levels of survivin and cyclin D1 in gastric carcinoma. Int J Oncol 2015;46:233-42.

30. Kumari S, Puneet, Prasad SB, et al. Cyclin D1 and cyclin E2 are differentially expressed in gastric cancer. Med Oncol 2016;33:40.

31. Chia J, Goh G, Bard F. Short O-GalNAc glycans: regulation and role in tumor development and clinical perspectives. Biochim Biophys Acta 2016;1860:1623-39.

32. Tamura F, Sato Y, Hirakawa M, et al. RNAi-mediated gene silencing of ST6GalNAc I suppresses the metastatic 
potential in gastric cancer cells. Gastric Cancer 2016;19:85-97.

33. Santos SN, Junqueira MS, Francisco G, et al. O-glycan sialylation alters galectin-3 subcellular localization and decreases chemotherapy sensitivity in gastric cancer. Oncotarget 2016;7:83570-87.

34. Li S, Zhuang Z, Wu T, et al. Nicotinamide nucleotide transhydrogenase-mediated redox homeostasis promotes tumor growth and metastasis in gastric cancer. Redox Biol 2018;18:246-55.

35. Beauchemin N, Arabzadeh A. Carcinoembryonic antigenrelated cell adhesion molecules (CEACAMs) in cancer progression and metastasis. Cancer Metastasis Rev 2013;32:643-71.

36. Takeuchi A, Yokoyama S, Nakamori M, et al. Loss of CEACAM1 is associated with poor prognosis and peritoneal dissemination of patients with gastric cancer. Sci Rep 2019;9:12702.

37. Judd LM, Bredin K, Kalantzis A, et al. STAT3 activation regulates growth, inflammation, and vascularization in a mouse model of gastric tumorigenesis. Gastroenterology 2006;131:1073-85.

38. Yu Z, Li Z, Wang C, et al. Oncostatin $M$ receptor, positively regulated by SP1, promotes gastric cancer growth and metastasis upon treatment with Oncostatin M. Gastric Cancer 2019;22:955-66.

39. Kurashige J, Hasegawa T, Niida A, et al. Integrated Molecular Profiling of Human Gastric Cancer Identifies DDR2 as a Potential Regulator of Peritoneal Dissemination. Sci Rep 2016;6:22371.

40. Kawaguchi T, Yan L, Qi Q, et al. Overexpression of suppressive microRNAs, miR-30a and miR-200c are associated with improved survival of breast cancer patients. Sci Rep 2017;7:15945.

41. Shimura T, Toden S, Kandimalla R, et al. Genomewide Expression Profiling Identifies a Novel miRNA-Based Signature for the Detection of Peritoneal Metastasis in Patients With Gastric Cancer. Ann Surg 2019. [Epub ahead of print].

42. Tseng CW, Lin CC, Chen CN, et al. Integrative network analysis reveals active microRNAs and their functions in gastric cancer. BMC Syst Biol 2011;5:99.

43. Deng M, Tang HL, Lu XH, et al. miR-26a suppresses tumor growth and metastasis by targeting FGF9 in gastric cancer. PLoS One 2013;8:e72662.

44. Qiu X, Zhu H, Liu S, et al. Expression and prognostic value of microRNA-26a and microRNA-148a in gastric cancer. J Gastroenterol Hepatol 2017;32:819-27.
45. Gong J, Li J, Wang Y, et al. Characterization of microRNA-29 family expression and investigation of their mechanistic roles in gastric cancer. Carcinogenesis 2014;35:497-506.

46. Bai F, Jiu M, You Y, et al. miR29a3p represses proliferation and metastasis of gastric cancer cells via attenuating HAS3 levels. Mol Med Rep 2018;17:8145-52.

47. Gu J, Li X, Li H, et al. MicroRNA-198 inhibits proliferation and induces apoptosis by directly suppressing FGFR1 in gastric cancer. Biosci Rep 2019. doi: 10.1042/ BSR20181258.

48. Zhao M, Liu Q, Liu W, et al. MicroRNA140 suppresses Helicobacter pyloripositive gastric cancer growth by enhancing the antitumor immune response. Mol Med Rep 2019;20:2484-92.

49. Zhu C, Huang Q, Zhu H. miR-383 Inhibited the Cell Cycle Progression of Gastric Cancer Cells via Targeting Cyclin E2. DNA Cell Biol 2019;38:849-56.

50. Chen D, Guo W, Qiu Z, et al. MicroRNA-30d-5p inhibits tumour cell proliferation and motility by directly targeting CCNE2 in non-small cell lung cancer. Cancer Lett 2015;362:208-17.

51. Liu Y, Zhou Y, Gong X, et al. MicroRNA-30a-5p inhibits the proliferation and invasion of gastric cancer cells by targeting insulin-like growth factor 1 receptor. Exp Ther Med 2017;14:173-80.

52. Sun F, Yu M, Yu J, et al. miR-338-3p functions as a tumor suppressor in gastric cancer by targeting PTP1B. Cell Death Dis 2018;9:522.

53. Ma DH, Li BS, Liu JJ, et al. miR-93-5p/IFNAR1 axis promotes gastric cancer metastasis through activating the STAT3 signaling pathway. Cancer Lett 2017;408:23-32.

54. Virgilio E, Giarnieri E, Giovagnoli MR, et al. Gastric Juice MicroRNAs as Potential Biomarkers for Screening Gastric Cancer: A Systematic Review. Anticancer Res 2018;38:613-6.

55. Yun J, Han SB, Kim HJ, et al. Exosomal miR-181b-5p Downregulation in Ascites Serves as a Potential Diagnostic Biomarker for Gastric Cancer-associated Malignant Ascites. J Gastric Cancer 2019;19:301-14.

56. Link A, Kupcinskas J. MicroRNAs as non-invasive diagnostic biomarkers for gastric cancer: Current insights and future perspectives. World J Gastroenterol 2018;24:3313-29.

57. Necula L, Matei L, Dragu D, et al. Recent advances in gastric cancer early diagnosis. World J Gastroenterol 2019;25:2029-44.

58. Hu Y, Qi C, Liu X, et al. Malignant ascites-derived 
exosomes promote peritoneal tumor cell dissemination and reveal a distinct miRNA signature in advanced gastric cancer. Cancer Lett 2019;457:142-50.

59. Guilliams M, Ginhoux F, Jakubzick C, et al. Dendritic cells, monocytes and macrophages: a unified nomenclature based on ontogeny. Nat Rev Immunol 2014;14:571-8.

60. Bagheri V, Abbaszadegan MR, Memar B, et al. Induction of $\mathrm{T}$ cell-mediated immune response by dendritic cells pulsed with mRNA of sphere-forming cells isolated from patients with gastric cancer. Life Sci 2019;219:136-43.

61. Kuramoto Y, Hashida M. Immunotherapy against peritoneal dissemination by immunostimulatory $\mathrm{CpG}$ DNA. Yakugaku Zasshi 2007;127:1533-40.

62. Räihä MR, Puolakkainen PA. Tumor-associated macrophages (TAMs) as biomarkers for gastric cancer: A review. Chronic Dis Transl Med 2018;4:156-63.

63. Wang X, Jiao X, Meng Y, et al. Methionine enkephalin (MENK) inhibits human gastric cancer through regulating tumor associated macrophages (TAMs) and PI3K/ AKT/mTOR signaling pathway inside cancer cells. Int Immunopharmacol 2018;65:312-22.

64. Seledtsov VI, Goncharov AG, Seledtsova GV. Clinically feasible approaches to potentiating cancer cellbased immunotherapies. Hum Vaccin Immunother 2015;11:851-69.

65. Scodeller P, Simon-Gracia L, Kopanchuk S, et al. Precision Targeting of Tumor Macrophages with a CD206 Binding Peptide. Sci Rep 2017;7:14655.

66. Harada K, Dong X, Estrella JS, et al. Tumor-associated macrophage infiltration is highly associated with PDL1 expression in gastric adenocarcinoma. Gastric Cancer 2018;21:31-40.

67. Muntasell A, Ochoa MC, Cordeiro L, et al. Targeting NK-cell checkpoints for cancer immunotherapy. Curr Opin Immunol 2017;45:73-81.

68. Du Y, Wei Y. Therapeutic Potential of Natural Killer Cells in Gastric Cancer. Front Immunol 2019;9:3095.

69. Tanaka H, Yashiro M, Sunami T, et al. ICAM-2 gene therapy for peritoneal dissemination of scirrhous gastric carcinoma. Clin Cancer Res 2004;10:4885-92.

70. Li M, Zheng H, Duan Z, et al. Promotion of cell proliferation and inhibition of ADCC by cancerous immunoglobulin expressed in cancer cell lines. Cell Mol Immunol 2012;9:54-61.

71. Wing JB, Sakaguchi S. Multiple treg suppressive modules and their adaptability. Front Immunol 2012;3:178.

72. Hu Z, Hu S, Wu Y, et al. Accumulation and suppressive function of regulatory $T$ cells in malignant ascites:
Reducing their suppressive function using arsenic trioxide in vitro. Oncol Lett 2018;15:5384-90.

73. Bagley SJ, O'Rourke DM. Clinical investigation of CAR T cells for solid tumors: lessons learned and future directions. Pharmacol Ther 2020;205:107419.

74. Liu X, Sun M, Yu S, et al. Potential therapeutic strategy for gastric cancer peritoneal metastasis by NKG2D ligandsspecific T cells. Onco Targets Ther 2015;8:3095-104.

75. Zhao W, Zhu H, Zhang S, et al. Trop2 is overexpressed in gastric cancer and predicts poor prognosis. Oncotarget 2016;7:6136-45.

76. Saito H, Kono Y, Murakami Y, et al. Highly Activated PD-1/PD-L1 Pathway in Gastric Cancer with PD-L1 Expression. Anticancer Res 2018;38:107-12.

77. Zhao $W$, Jia L, Zhang $M$, et al. The killing effect of novel bi-specific Trop2/PD-L1 CAR-T cell targeted gastric cancer. Am J Cancer Res 2019;9:1846-56.

78. Kawazoe A, Shitara K, Kuboki Y, et al. Clinicopathological features of 22C3 PD-L1 expression with mismatch repair, Epstein-Barr virus status, and cancer genome alterations in metastatic gastric cancer. Gastric Cancer 2019;22:69-76.

79. Yu Y, Ma X, Zhang Y, et al. Changes in Expression of Multiple Checkpoint Molecules and Infiltration of Tumor Immune Cells after Neoadjuvant Chemotherapy in Gastric Cancer. J Cancer 2019;10:2754-63.

80. Wang Z, Yin N, Zhang Z, et al. Upregulation of T-cell Immunoglobulin and Mucin-Domain Containing-3 (Tim3 ) in Monocytes/Macrophages Associates with Gastric Cancer Progression. Immunol Invest 2017;46:134-48.

81. Chou FC, Chen HY, Kuo CC, et al. Role of Galectins in Tumors and in Clinical Immunotherapy. Int J Mol Sci 2018. doi: 10.3390/ijms19020430.

82. Wang Y, Zhao E, Zhang Z, et al. Association between Tim3 and Gal9 expression and gastric cancer prognosis. Oncol Rep 2018;40:2115-26.

83. Liao W, Lin JX, Leonard WJ. Interleukin-2 at the crossroads of effector responses, tolerance, and immunotherapy. Immunity 2013;38:13-25.

84. Sun T, Zhou Y, Yang M, et al. Functional genetic variations in cytotoxic T-lymphocyte antigen 4 and susceptibility to multiple types of cancer. Cancer Res 2008;68:7025-34.

85. Lim HS, Cordoba SP, Dushek O, et al. Costimulation of IL-2 Production through CD28 Is Dependent on the Size of Its Ligand. J Immunol 2015;195:5432-9.

86. Kosaka K, Yashiro M, Sakate Y, et al. A synergistic antitumor effect of interleukin-2 addition with CD80 immunogene therapy for peritoneal metastasis of gastric carcinoma. Dig Dis Sci 2007;52:1946-53. 
87. Bhandaru M, Rotte A. Monoclonal Antibodies for the Treatment of Melanoma: Present and Future Strategies. Methods Mol Biol 2019;1904:83-108.

88. Bennett F, Luxenberg D, Ling V, et al. Program death-1 engagement upon TCR activation has distinct effects on costimulation and cytokine-driven proliferation: attenuation of ICOS, IL-4, and IL-21, but not CD28, IL7, and IL-15 responses. J Immunol 2003;170:711-8.

89. Iwamura T, Narumi H, Suzuki T, et al. Novel pegylated interferon-beta as strong suppressor of the malignant ascites in a peritoneal metastasis model of human cancer. Cancer Sci 2017;108:581-9.

90. Wang T, Wei Y, Tian L, et al. C-C motif chemokine ligand
5 (CCL5) levels in gastric cancer patient sera predict occult peritoneal metastasis and a poorer prognosis. Int J Surg 2016;32:136-42.

91. Luo J, Chen XQ, Li P. The Role of TGF-beta and Its Receptors in Gastrointestinal Cancers. Transl Oncol 2019;12:475-84.

92. Katz LH, Likhter M, Jogunoori W, et al. TGF-beta signaling in liver and gastrointestinal cancers. Cancer Lett 2016;379:166-72.

93. Lv ZD, Zhao WJ, Jin LY, et al. Blocking TGF-beta1 by $\mathrm{P} 17$ peptides attenuates gastric cancer cell induced peritoneal fibrosis and prevents peritoneal dissemination in vitro and in vivo. Biomed Pharmacother 2017;88:27-33. doi: $10.21037 /$ tgh.2020.02.08

Cite this article as: Yao X, Ajani JA, Song S. Molecular biology and immunology of gastric cancer peritoneal metastasis. Transl Gastroenterol Hepatol 2020;5:57. 